\title{
Single cell transcriptomics of neighboring hyphae of Aspergillus niger
}

Charissa de Bekker ${ }^{1}$, Oskar Bruning ${ }^{2}$, Martijs J Jonker ${ }^{2,3}$, Timo M Breit ${ }^{2,3}$ and Han AB Wösten ${ }^{1 *}$

\begin{abstract}
Single cell profiling was performed to assess differences in RNA accumulation in neighboring hyphae of the fungus Aspergillus niger. A protocol was developed to isolate and amplify RNA from single hyphae or parts thereof. Microarray analysis resulted in a present call for 4 to $7 \%$ of the A. niger genes, of which $12 \%$ showed heterogeneous RNA levels. These genes belonged to a wide range of gene categories.
\end{abstract}

\section{Background}

Cellular heterogeneity within an isogenic cell population is a widespread event in both prokaryotic and eukaryotic organisms. Heterogeneity of cells can be beneficial for the organism in many ways. Many documented cases of phenotypic variability in microorganisms relate to responses to environmental stress. This suggests that phenotypic variation aids in the survival of cells under adverse conditions and therefore may be an evolvable trait $[1,2]$. It has been shown that mycelia of filamentous fungi are also heterogeneous. For instance, protein secretion [3-5] and gene expression [6-9] are heterogeneous between zones of fungal colonies. These differences were explained by the availability of carbon source and by spatial and temporal differentiation [7]. Heterogeneous gene expression can even be found within a zone of a colony. In fact, expression of the glucoamylase gene glaA, the acid amylase gene aamA, the $\alpha$-glucuronidase gene $\operatorname{ag} u A$, and the feruloyl esterase gene faeA is heterogeneous between neighboring hyphae at the periphery of the colony of Aspergillus niger $[10,11]$. Co-expression studies showed that hyphae that highly express one of these genes also highly express the other genes encoding secreted proteins [11]. Moreover, these hyphae highly express the glyceraldehyde-3-phosphate dehydrogenase gene $g p d A$, and are characterized by a high $18 S$ rRNA content. Taken together, it was concluded that at least two subpopulations of hyphae

\footnotetext{
* Correspondence: h.a.b.wosten@uu.nl

${ }^{1}$ Microbiology and Kluyver Centre for Genomics of Industrial Fermentations, Institute of Biomembranes, Utrecht University, Padualaan 8, 3584 CH Utrecht, The Netherlands

Full list of author information is available at the end of the article
}

exist within the outer zone of the mycelium of $A$. niger. These subpopulations are characterized by a high and a low transcriptional activity, respectively [11]. The data implied also that the translational activity may be different in the two populations of hyphae.

Transcriptome analysis of single cells is an important tool to understand the extent of cellular heterogeneity and its underlying mechanisms. So far, whole genome expression analysis has been reported of an individual neuron and a single blastomere [12,13]. Here, we performed for the first time a single cell transcriptome analysis in a microbe. It is shown that the RNA composition of neighboring hyphae at the periphery of an $A$. niger mycelium is heterogeneous. Heterogeneity can be found in all functional gene classes (FunCats) as well as in rRNAs and tRNAs.

\section{Results}

Hyphal architecture at the periphery of a sandwiched colony

Distribution of nuclei and septa was monitored at the periphery of 7-day-old sandwiched colonies of $A$. niger using a fusion of the histone $\mathrm{H} 2 \mathrm{~B}$ protein and green fluorescent protein (H2B-GFP fusion) and calcofluor white, respectively. Septa were not detected within the first $400 \mu \mathrm{m}$ from the tip (Figure 1a). After the first septum, septa were separated by 50 to $100 \mu \mathrm{m}$. Nuclei were found throughout the hypha, except for the region 10 to $20 \mu \mathrm{m}$ from the tip (Figure 1b, c). Taken together, only part of the first compartment of hyphae of $A$. niger is analyzed when tip regions of 100 to $200 \mu \mathrm{m}$ are dissected for RNA analysis (see below). 


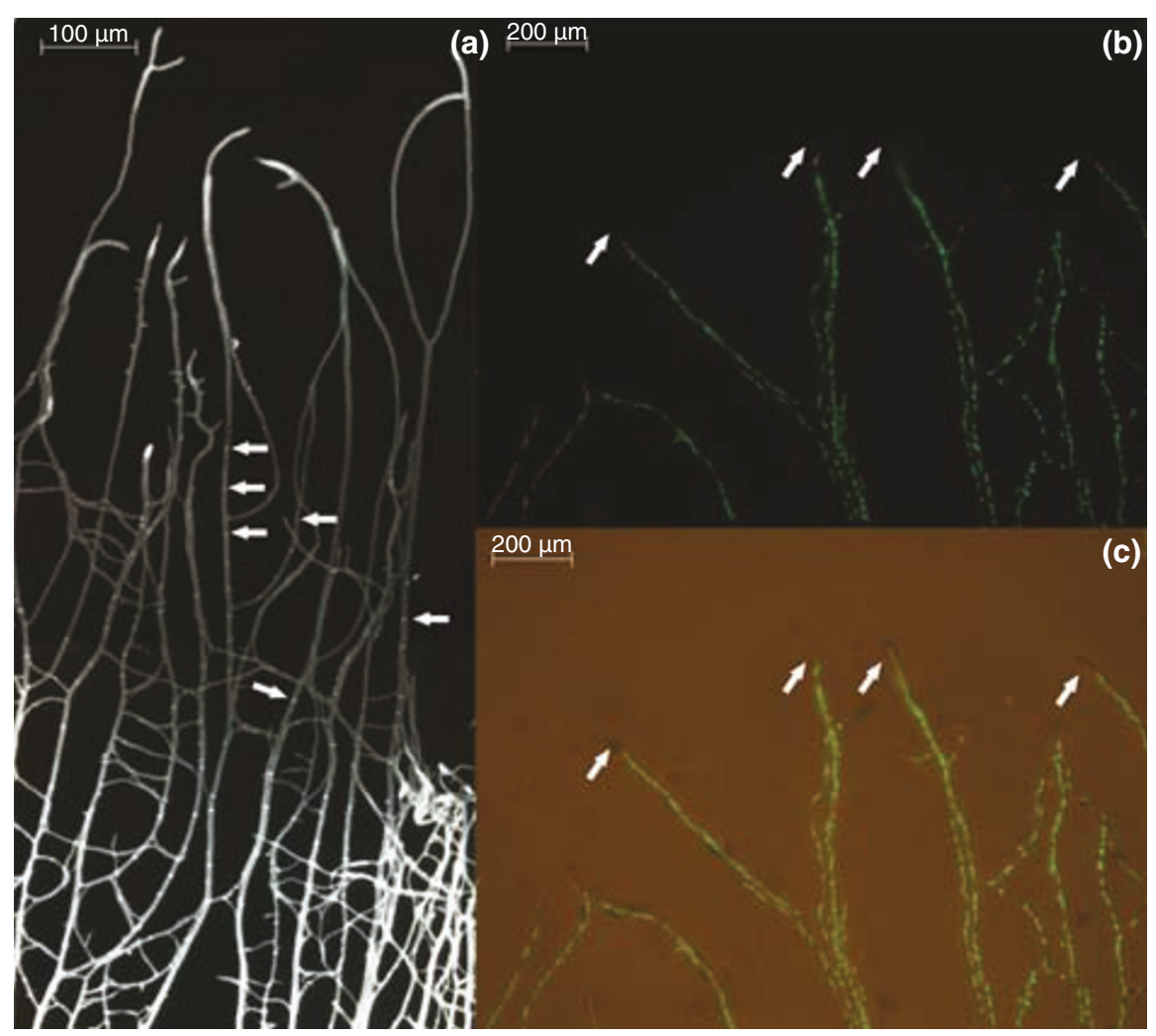

Figure 1 Distribution of septa and nuclei in hyphae at the outer part of a sandwiched colony. (a) Calcofluor white staining visualizing the septa within the hyphae (indicated by arrows). The first septum is positioned $400 \mu \mathrm{m}$ from the apex of the hyphae. (b) GFP fused to H2B visualizing the nuclei. (c) Overlay of (b) with a bright field image. The region 10 to $20 \mu \mathrm{m}$ from the apex is free from nuclei (indicated by arrows).

\section{RNA profiling of single hyphal tips}

A reproducible RNA extraction and amplification protocol was developed to enable analysis of transcript profiles of selected (parts of) hyphae within a mycelium (Additional file 1). This protocol includes growth conditions and sample preparation, laser dissection, RNA isolation, and cDNA amplification and labeling. The protocol was used to isolate RNA from 1,000 hyphal tips (with a width of 3 to $4 \mu \mathrm{m}$ and a length of $100 \mu \mathrm{m}$ ) from the outer periphery of 7-day-old sandwiched colonies of A. niger strain AR9\#2. The RNA was spotted onto a nylon membrane and hybridized with an $18 S$ rDNA probe. The hybridization signal was compared to that of samples with a known RNA concentration. From this it was concluded that 1,000 hyphal tips with a length of $100 \mu \mathrm{m}$ contain 1 ng of RNA (Additional file 2).

RNA was isolated from five single tips with a length of $200 \mu \mathrm{m}$ of neighboring hyphae from the outermost region of a 7-day-old $A$. niger sandwiched colony. To this end, fragments of each hypha were catapulted into a cap of an Eppendorf tube using the autoLPC option (Figure 2a-c). After RNA isolation, half of the total RNA contained in each of the five samples was converted into
cDNA. This cDNA was amplified to 5.9 to $10.1 \mu \mathrm{g}$ with the WT-Ovation One-Direct RNA Amplification System (Nugen, San Carlos, CA, USA) and used for quantitative PCR (QPCR) and hybridization of Affymetrix A. niger gene chips (Affymetrix, Santa Clara, CA, USA). The amplicons of three of the samples (hyphae 1 to 3 ) were mainly 50 to $100 \mathrm{bp}$ in length, while most of the amplicons of the other two hyphae (hyphae 4 and 5) had a length of 100 to $300 \mathrm{bp}$ (Figure 2d). Notably, the latter two samples had been amplified on a different day than the former three samples.

\section{Hyphal heterogeneity analyzed by QPCR}

The amplified cDNA samples of the five single hyphae were analyzed by QPCR. As a control, amplified cDNA was used from three biological replicates of a pool of 100 hyphal tips and of mycelium of the whole periphery of sandwiched colonies. Cycle threshold (Ct) levels were determined for $18 S$ rRNA, actin, and glaA using $1 \mathrm{ng}$ cDNA and six technical replicates for each sample. Ct values for the RNA samples from the periphery of the colony were very similar (Table 1). An F-test showed that the standard deviation of the biological replicates 

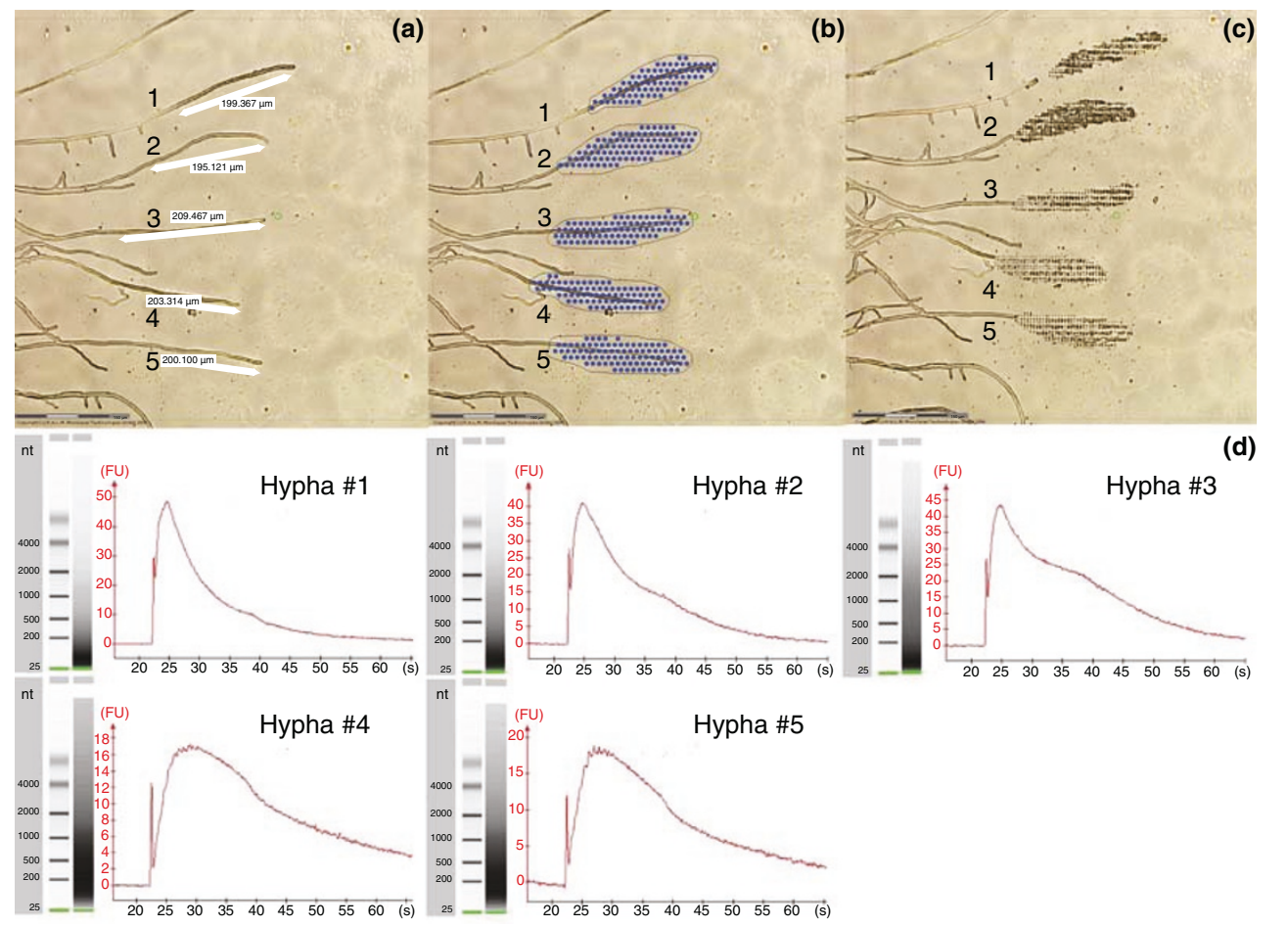

Figure 2 Amplification of cDNA from the tips of five single neighboring hyphae. (a-c) Apical regions of 200 um of hyphae at the periphery of sandwiched colonies were selected by using the measuring (a) and drawing (b) tool. Fragments of a single hypha were catapulted into a cap of an Eppendorf tube by using the autoLPC option (c). RNA of the single hyphae was isolated, converted into cDNA and amplified. (d) The amplified cDNA was analyzed with a Bioanalyser. The electrophoresis gel image (nt, nucleotides; L, ladder) and the electropherogram (yaxis represents fluorescence units (FU); $x$-axis represents run time in seconds (s)) are given for the five samples. Amplicons of hyphae 1 to 3 and hyphae 4 and 5 were mainly 50 to $100 \mathrm{bp}$ and 100 to $300 \mathrm{bp}$ in length, respectively.

was not significantly higher than the maximum standard deviation obtained for one of the series of technical triplicates $(P \leq 0.01$; Additional file 3$)$. Standard deviations found for the $\mathrm{Ct}$ values of the samples of 100 hyphal

Table 1 Accumulation of RNA is heterogeneous between hyphae at the periphery of an A.niger colony

\begin{tabular}{llccc}
\hline Gene & Sample type & $\boldsymbol{\mu C t}$ & $\boldsymbol{\sigma}$ & $\boldsymbol{\sigma}$ technical \\
\hline 185 & 1 hypha & 20.23 & 2.40 & $0.53-1.26$ \\
& 100 hyphae & 17.39 & 0.42 & $0.26-0.43$ \\
\multirow{4}{*}{ actin } & 5 pg periphery & 12.58 & 0.28 & $0.15-0.35$ \\
& 1 hypha & 30.30 & 6.07 & $0.11-1.03$ \\
& 100 hyphae & 28.15 & 4.80 & $0.12-1.71$ \\
glaA & 5 pg periphery & 18.05 & 0.57 & $0.09-0.22$ \\
& 1 hypha & 25.47 & 4.63 & $0.06-0.66$ \\
& 100 hyphae & 24.51 & 2.22 & $0.10-0.22$ \\
& 5 pg periphery & 18.65 & 0.49 & $0.10-0.14$ \\
\hline
\end{tabular}

QPCR was performed using $1 \mathrm{ng}$ of cDNA amplified from RNA of tips of single neighboring hyphae from the outer periphery of a sandwiched colony, from RNA of a pool of 100 of such tips, and from RNA of the 3-mm-wide periphery of sandwiched colonies of $A$. niger. The average cycle threshold $(\mu \mathrm{Ct})$ and their standard deviations $(\sigma)$ are given for $18 \mathrm{~S}$ rDNA and the actin and glaA genes. $\sigma$ technical represents the range in standard deviations obtained for the six technical replicates for each of the biological replicates. tips were between 1.5 and 8.4 fold higher when compared to the cDNA from the whole periphery but they were not significantly different from the technical replicates $(P \leq 0.01$; Table 1 ; Additional file 3$)$. The differences in RNA levels were even more pronounced when individual hyphae were compared (Table 1; Additional file 3). The standard deviations for the $\mathrm{Ct}$ values of the glaA and actin genes of hyphae 1 to 5 were significantly higher when compared to the maximum standard deviation obtained for the technical replicates within this sample type. This difference was not observed with the $18 S$ rRNA gene. Similar results were obtained with the standard deviation of the levels of $18 S$ rRNA, and glaA and actin mRNA when hyphae 1 to 3 and hyphae 4 and 5 were analyzed separately (Additional file 3 ). This shows that the differences in RNA levels are not due to a batch effect.

\section{Hyphal heterogeneity analyzed by microarrays}

Biotin-labeled amplified cDNA of the single hyphal tips was hybridized to Affymetrix A. niger gene chips. Based on MAS5.0 detection calls, transcripts of 4.1 to $6.7 \%$ of the genes had a present call in each of the single hyphae 
(Additional file 4). Genes with an absent call had generally low signal values in hybridization experiments where 500 pg RNA from the periphery or from a pool of 500 hyphal tips was used [14] (Additional file 5). The scale factors of the sample types had a difference $<5$ fold. Due to the low number of present calls, this difference in scale factors was considered to be low enough to normalize and analyze the samples as a whole. In total, 2,608 of the 14,455 probe sets had a present call in at least one of the samples of the single hyphae (Additional file 6). These probe sets were found to comprise all 19 different class I functional categories (FunCats) as well as the non-FunCat categories tRNA and rRNA. Almost half of the detectable probe sets belonged to unclassified proteins (Table 2). Metabolism was the second largest group, with 550 hybridizing probe sets. Categories with more than 50 probe sets with a present call comprised protein fate (148), transcription (116), cell cycle and DNA processing (106), cellular transport and transport mechanisms (76), protein synthesis (75), and cell rescue, defense and virulence (52). The categories with a lower number of present calls generally comprised a small number of total probe sets. In most categories 10 to $30 \%$ of the probe sets had a present call. This was 50 to $75 \%$ for the categories rRNA, tissue localization, and protein with binding function or cofactor requirement. Similar results for the FunCat analysis were obtained when hyphae 1 to 3 and hyphae 4 and 5 were analyzed separately (data not shown). This shows that the analysis was hardly, if at all, affected by a batch effect.

Hierarchical clustering of the hyphae was done on basis of the Z-scores of the $\log 2$ signals of the robust multiarray analysis (RMA) using the 2,608 probe sets that had a present call in at least one of the hyphae (Figure 3a). As a distance (d) measure 1-the Pearson correlation was used (a distance of 0 means that the samples are identical; a distance of 2 means the samples are completely different). Hyphae 1 and 2 were least distant $(\mathrm{d}=$ 1.10). The correlation between hypha 3 and hyphae 1 and 2 and between hyphae 4 and 5 was similar $(\mathrm{d}=$ 1.22 and $d=1.24$, respectively). Principal component analysis (PCA) revealed that hypha 4 was separated from the other samples in the first principal component. Hypha 5 was separated in the second principal component, whereas hyphae 2 and 3 were separated in the third principal component (Figure 3b). In the next analysis, overrepresentation of functional gene categories

Table 2 Classification of probe sets with a present call in at least one of the five arrays of a single hypha

\begin{tabular}{|c|c|c|c|}
\hline Category & $\begin{array}{l}\text { Number of probe sets with a } \\
\text { present call }\end{array}$ & $\begin{array}{c}\text { Total number of probe } \\
\text { sets }\end{array}$ & $\begin{array}{l}\text { Percentage of probe sets } \\
\text { present }\end{array}$ \\
\hline 01 Metabolism & 550 & 3023 & 18.2 \\
\hline 02 Energy & 25 & 117 & 21.4 \\
\hline 03 Cell cycle and dna processing & 106 & 476 & 22.3 \\
\hline 04 Transcription & 116 & 715 & 16.2 \\
\hline 05 Protein synthesis & 75 & 241 & 31.1 \\
\hline 06 Protein fate (folding, modification, destination) & 148 & 616 & 24 \\
\hline 08 Cellular transport and transport mechanisms & 76 & 438 & 17.4 \\
\hline $\begin{array}{l}10 \text { Cellular communication or signal transduction } \\
\text { mechanism }\end{array}$ & 31 & 179 & 17.3 \\
\hline 11 Cell rescue, defense and virulence & 52 & 267 & 19.5 \\
\hline $\begin{array}{l}13 \text { Regulation of or interaction with cellular } \\
\text { environment }\end{array}$ & 8 & 73 & 11 \\
\hline 14 Cell fate & 15 & 104 & 14.4 \\
\hline 25 Development (systemic) & 6 & 26 & 23.1 \\
\hline $\begin{array}{l}29 \text { Transposable elements, viral and plasmid } \\
\text { proteins }\end{array}$ & 12 & 62 & 19.4 \\
\hline 30 Control of cellular organization & 5 & 29 & 17.2 \\
\hline 40 Sub-cellular localization & 19 & 137 & 13.9 \\
\hline 45 Tissue localization & 1 & 2 & 50 \\
\hline $\begin{array}{l}63 \text { Protein with binding function or co-factor } \\
\text { requirement }\end{array}$ & 1 & 2 & 50 \\
\hline 67 Transport facilitation & 5 & 44 & 11.4 \\
\hline 99 Unclassified proteins & 1,279 & 7,670 & 16.7 \\
\hline rRNA & 6 & 8 & 75 \\
\hline tRNA & 39 & 144 & 27.1 \\
\hline
\end{tabular}

Classification of the 2,608 probe sets is based on class I FunCats as well as the non-FunCat categories tRNA and rRNA. 

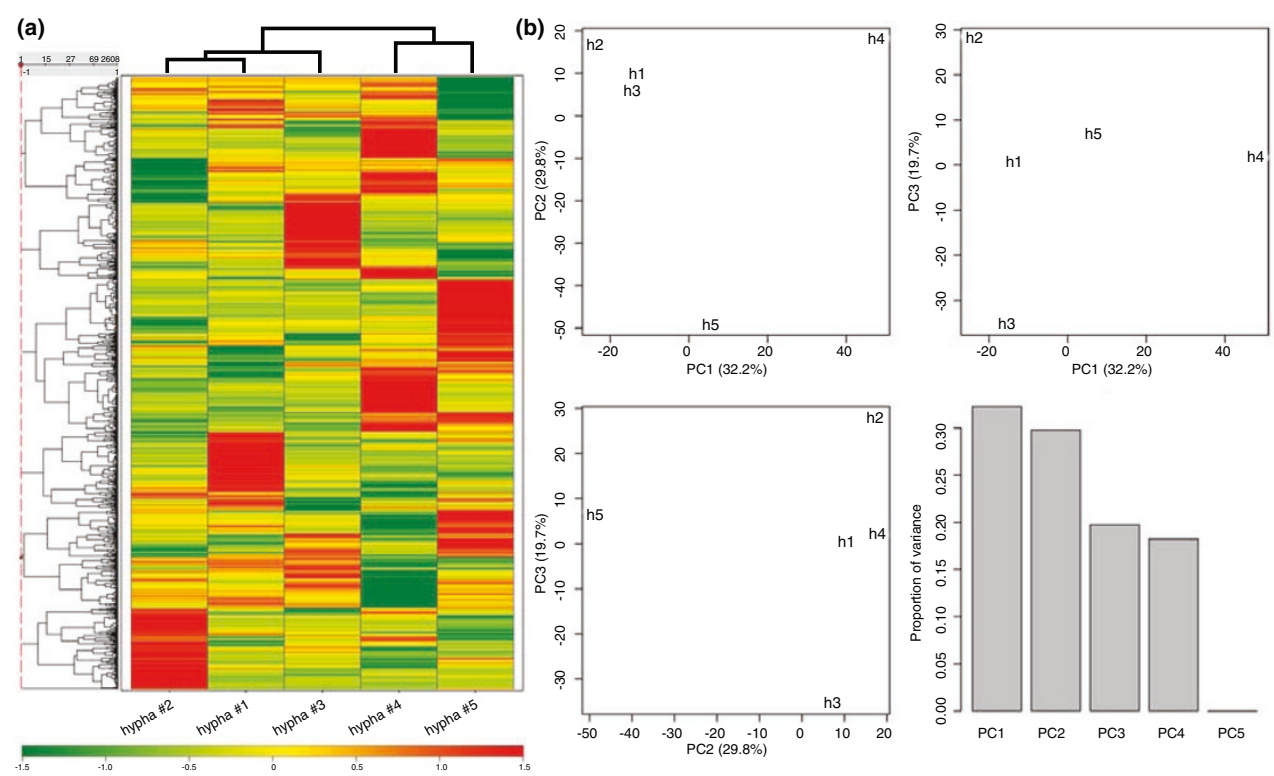

Figure 3 Hierarchal clustering and principal component analysis of RNA profiles of five neighboring hyphae. (a, b) Hierarchal clustering (a) and principal component analysis (PCA) (b) were done on the basis of the 2,608 probe sets that had a present call in at least one of the single hyphae. Clustering was done using complete linkage as clustering method with 1-correlation as distance measure. The Z-scores of the $\log 2$ RMA signal values of the 2,608 probe sets were used for clustering and PCA.

was tested for all probe sets with a present call in each of the individual hyphae. This revealed that the nonFunCat categories rRNA and tRNA were overrepresented in all five hyphae (Table 3; Additional file 7). Ribosome biogenesis was overrepresented in four hyphae, whereas other export and secretion systems and proteolytic degradation were overrepresented in two of the hyphae. Similar results for the over-representation analysis were obtained when hyphae 1 to 3 and hyphae 4 and 5 were analyzed separately (data not shown). This shows that the analysis was hardly, if at all, affected by a batch effect.
Within the 2,608 probe sets with a present call in at least one of the five single hyphae, 308 showed a relatively high standard deviation ( $>0.5$ ) between the $\log 2$ RMA signal values (Additional file 8 ). Within these probe sets, 5 out of 19 class I FunCats are not represented. These five categories (regulation of or interaction with cellular environment, development, tissue localization, protein with binding function or co-factor requirement, and transport facilitation) have relatively few members (with a maximum of 73 members). The hyphae were clustered based on the Zscores of the signals of the 308 probe sets (Figure 4a).

Table 3 Overrepresented functional gene categories within the set of genes with a present call in a single hypha

\begin{tabular}{|c|c|c|c|c|c|c|}
\hline \multirow[b]{2}{*}{ Functional gene category } & \multirow[t]{2}{*}{ Number of probe sets } & \multicolumn{5}{|c|}{ Hypha number ${ }^{a}$} \\
\hline & & 1 & 2 & 3 & 4 & 5 \\
\hline 03.01.03 DNA synthesis and replication & 96 & 1 & 0 & 0 & 0 & 0 \\
\hline 03.01 DNA processing & 240 & 1 & 0 & 0 & 0 & 0 \\
\hline 05.01 Ribosome biogenesis & 138 & 1 & 1 & 1 & 0 & 1 \\
\hline 06.13.04 Lysosomal and vacuolar degradation & 32 & 0 & 0 & 1 & 0 & 0 \\
\hline 06.13 Proteolytic degradation & 198 & 0 & 1 & 1 & 0 & 0 \\
\hline 08.16.99 Other export and secretion systems & 15 & 0 & 0 & 1 & 1 & 0 \\
\hline 29.01 LTR retro-elements (retro-viral) & 28 & 0 & 0 & 1 & 0 & 0 \\
\hline 40.05 Centrosome & 9 & 0 & 0 & 0 & 1 & 0 \\
\hline 67.04.01.02 Other cation transporters (NA, K, CA, NH4, etc.) & 33 & 0 & 0 & 0 & 1 & 0 \\
\hline rRNA & 8 & 1 & 1 & 1 & 1 & 1 \\
\hline tRNA & 144 & 1 & 1 & 1 & 1 & 1 \\
\hline
\end{tabular}

${ }^{\mathrm{a}} 1$, overrepresentation of a category $(P<0.01) ; 0$, absence of overrepresentation $(P>0.01)$. For $P$-values see Additional file 7 . 

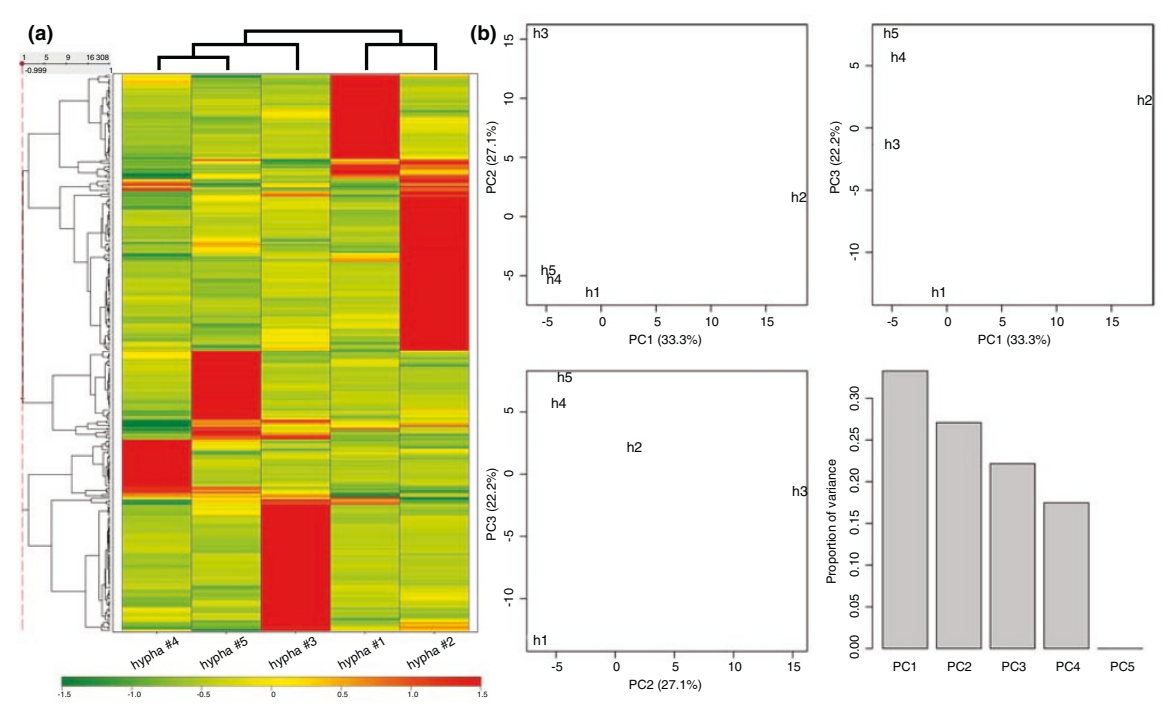

Figure 4 Hierarchal clustering and PCA of RNA profiles of five neighboring hyphae. (a, b) Hierarchal clustering (a) and PCA (b) were done on the basis of the 308 probe sets that had a present call in at least one out of five hyphae and that showed a standard deviation $>0.5$ between the signal values. Clustering was done using complete linkage as clustering method with 1-correlation as distance measure. The Zscores of the log2 RMA signal values of the 308 probe sets were used for clustering and PCA.

This revealed that hyphae 4 and 5 were most similar. Hypha 3 was more similar to hyphae 4 and 5 than to hyphae 1 and 2. PCA (Figure $4 \mathrm{~b}$ ) revealed that in the first principal component, hypha 2 separated from the other samples, whereas hypha 3 and hypha 1 were separated in the second and third principal components, respectively. Each hypha showed a cluster of genes with higher signals when compared to the other four hyphae (Figure 4a). These clusters were analyzed for overrepresentation of functional gene categories (Table 4; Additional file 7). This revealed that the classes ribosome biogenesis and tRNA were overrepresented in three of the five hyphae. The cluster of hypha 5 was not enriched in any functional category.
In contrast, seven FunCats were overrepresented in the cluster of hypha 2, among which two are involved in energy. Separate analysis of hyphae 1 to 3 and hyphae 4 and 5 had an effect on the over-representation analysis. As mentioned above, the analysis was based on a list of highly variable genes with a present call in at least one sample. The results of the analysis were not different because of a batch effect but simply because it was based on three (or two) instead of five samples. Indeed, very similar significance values were obtained for almost all functional gene categories in Table 4 when the five hyphae were analyzed together or when hyphae 1 to 3 and 4 and 5 were analyzed separately (Additional file 9).

Table 4 Overrepresented functional gene categories within the set of genes that have a signal value with a standard deviation of $>0.5$ between the five single hyphae

\begin{tabular}{|c|c|c|c|c|c|c|}
\hline \multirow[b]{2}{*}{ Functional gene category } & \multirow[t]{2}{*}{ Number of probe sets } & \multicolumn{5}{|c|}{ Hypha number $^{a}$} \\
\hline & & 1 & 2 & 3 & 4 & 5 \\
\hline 02.13.03 Aerobic respiration & 62 & 0 & 1 & 0 & 0 & 0 \\
\hline 02.13 Respiration & 93 & 0 & 1 & 0 & 0 & 0 \\
\hline 02.19 Metabolsim of energy reserves (for example, glycogen, trehalose) & 36 & 1 & 0 & 0 & 0 & 0 \\
\hline 04.05.01.01 General transcription activities & 107 & 0 & 1 & 0 & 0 & 0 \\
\hline 05.01 Ribosome biogenesis & 138 & 1 & 1 & 1 & 0 & 0 \\
\hline 06.07.99 Other protein modifications & 54 & 0 & 0 & 0 & 1 & 0 \\
\hline 06.13 Proteolytic degradation & 198 & 0 & 1 & 0 & 0 & 0 \\
\hline 30.10 Nucleus & 55 & 0 & 1 & 0 & 0 & 0 \\
\hline 67.15 Electron or hydrogen carrier & 48 & 0 & 1 & 0 & 0 & 0 \\
\hline tRNA & 144 & 1 & 0 & 1 & 1 & 0 \\
\hline
\end{tabular}

${ }^{a} 1$, overrepresentation of a category $(P<0.01) ; 0$, absence of overrepresentation $(P>0.01)$. For $P$-values see Additional file 7 . 
The top 100 genes with the highest hybridization signal in each of the hyphae was selected (Additional file 10). This selection comprised a total of 207 genes. Of these genes, 43 and 18 are found within the top 100 of all 5 hyphae and of 4 hyphae, respectively (Additional file 11). A major part of these genes (19 out of 43 and 11 out of 18) encode unidentified proteins. Examples of genes that have a predicted function are the 4 rRNAs, 4 tRNAs and 14 genes involved in metabolism. A number of 119 genes (of which 43 encode unidentified proteins) were found in only one of the hyphae (Additional file 12). The glucoamylase gene glaA, the cellulase gene eglb, 7 genes encoding cytoplasmic ribosomal proteins and 13 tRNAs were among these genes. The 119 genes were randomly distributed in the top 100 of the different hyphae.

\section{Discussion}

A disconnection has been observed between the average gene expression of a culture of isogenic cells and the gene expression of a single cell within such a culture $[15,16]$. Therefore, single cell analysis with high spatiotemporal resolution is needed to give an accurate understanding of the processes within a cell. Single cell analysis is not yet widely applied mainly because of the fact that the technologies that are needed are not fully developed. The fact that microbial cells are much smaller than those of plants and animals complicates the use of these technologies. Here, we developed a protocol for single cell transcriptome analysis of hyphae of the microbe $A$. niger. Using this protocol, it is shown that neighboring hyphae have a heterogeneous RNA composition despite the fact that they experience identical environmental conditions. Differences in RNA accumulation were shown to occur in all functional gene groups (FunCats) as well as in rRNAs and tRNAs. The observed fluctuations in RNA accumulation between individual hyphae are supported by GFP reporter studies and in situ hybridizations $[10,11,17]$. The variation in transcriptome composition is not the result of differences in growth rate. The hyphae that were selected had a similar diameter and extension rate (our unpublished data). Stochastic effects, chromatin folding, transcript transport/motility, and differences in timing of mitosis may have caused the fluctuations. H2A-GFP reporter studies indicated that the mitotic index of the leading hyphae is different. This was implied from the observation that the relative number of elongated GFP stained structures (representing nuclei that undergo mitotis) was different between the hyphae (Figure 1c). Differences in RNA composition may not only be related to cell cycledependent expression, it may also be due to a sharp exit of RNA from the nuclei during mitosis. This is suggested from the fact that nuclear pore complexes partially disassemble during mitosis in A. nidulans [18], thus abolishing the permeability barrier of the nuclear membrane as is found in other eukaryotes [19].

By hybridizing RNA from 1,000 hyphal tips with a $18 \mathrm{~S}$ rRNA probe, it was shown that the first $100 \mu \mathrm{m}$ of the tip region of exploring hyphae of $A$. niger contains $1 \mathrm{pg}$ of RNA. It is known that a typical mammalian cell contains about 10 to $30 \mathrm{pg}$ total RNA [20], whereas the smaller Escherichia coli cells contain about 5.6 fg RNA [21]. The amount of RNA in fungal hyphae or yeast cells was not yet established. Saccharomyces cerevisiae has been reported to contain 60,000 mRNAs per cell [22]. Assuming that these mRNAs comprise $5 \%$ of the total RNA [20,23] and that the average RNA length is 2,500 nucleotides [20], S. cerevisiae would contain 2.5 pg total RNA per cell. This amount is well in line with the $1 \mathrm{pg}$ of RNA that was extracted from the hyphal tip region of $A$. niger. The low amount of RNA within a cell requires amplification to microgram quantities to enable hybridization of DNA microarrays. We used the Ribo-SPIA Technology developed by Nugen. This technology gives the most reliable results when compared to other amplification protocols [24] (our own unpublished results). As a consequence of the Ribo-SPIA Technology, rRNA is also amplified.

RNA profiles were determined for the tip region of five neighboring hyphae at the most outer part of a colony of $A$. niger. This was done in two amplification experiments. The cDNA amplicons of one of the experiments had a length of 100 to $300 \mathrm{bp}$, whereas those of the other experiment were 50 to 100 bp in length. Hybridization of Affymetrix GeneChip A. niger Genome Arrays revealed that the higher amplicon length was accompanied by a lower number of genes with a present call (5.8 to $6.7 \%$ versus 4.1 to $4.3 \%$ for the small and large amplicons, respectively). The number of genes with a present call is low (4.1 to 6.7\%) when one considers that about $50 \%$ of the genes are expressed in a sandwiched colony of $A$. niger [7]. Genes that were lowly expressed at the periphery of the colony [7] or within a pool of hyphal tips from exploring hyphae [14] often had an absent call in the arrays of the single hyphae. Apparently, RNA of lowly expressed genes is not sufficiently amplified when one uses the RNA of a single hypha. As a consequence of the low number of probe sets with a present call, higher scale factors are obtained when compared to hybridization experiments with RNA from the whole colony. Furthermore, scale factors are influenced by minimal differences in the number of present calls between the samples. Taking these facts into account, the difference in scale factors between the arrays of the single hyphae (more than three-fold but less than five-fold) was considered to be low enough to normalize and analyze the samples as a whole using the 
RMA method to normalize between slides. Statistical analysis indicated that this was justified (see Materials and methods).

In total, 2,608 probe sets had a present call in at least one of the five individual hyphae. These probe sets were found to comprise tRNAs, rRNAs and all 19 class I FunCats. For each functional gene category, at least 10 to $30 \%$ of the probe sets had a present call, indicating that all categories were evenly well detected. Heterogeneity between the five individual exploring hyphae was assessed by testing for overrepresentation of functional gene categories within the pool of genes with a present call within each of the single hyphae. The test revealed that genes encoding rRNAs and tRNAs were overrepresented in all five hyphae, while genes involved in ribosome biogenesis were overrepresented in four out of the five hyphae. The other nine overrepresented categories were found in one or two of the five hyphae. Heterogeneity in RNA composition was also indicated by the finding that 308 out of the 2,608 probe sets had a relatively high standard deviation $(>0.5)$ of the $\log 2$ RMA signal values. Apparently, expression of at least $12 \%$ of the genes is heterogeneous between neighboring exploring hyphae. This set of genes comprises all functional gene categories, except for the ones that have relatively few members. Hierarchical clustering of the 308 probe sets showed that each single hypha had a cluster of genes with higher signals when compared to the other four hyphae. Ribosome biogenesis and tRNAs were overrepresented in the majority of the samples. One hypha showed no overrepresented categories, whereas another hypha showed overrepresentation for 7 of the 10 found categories. Two of these enriched categories were involved in energy, implying that this hypha might have been metabolically more active than the other hyphae. Heterogeneity within the five individual hyphae was also assessed by selecting the top 100 genes with the highest hybridization signal in each of the hyphae. A total of 207 different genes was found in this selection, of which 43 were found in all 5 hyphae and 119 were found exclusively in one of the hyphae. For instance, all 5 hyphae contained the 4 rRNAs $(5 S, 5.8 S, 18 S$ and 28S), 4 tRNAs and 14 genes involved in metabolism in their top 100. In contrast, 13 tRNAs, 7 genes encoding cytoplasmic ribosomal proteins and glaA and $e g l B$ were present in the top 100 of only one of the hyphae. The gene glaA and $18 S$ rRNA were also found in the list of 308 genes that showed a standard deviation $>0.5$ between the log2 RMA signal values. Heterogeneity in the RNA levels of these genes as well as the actin gene was confirmed by QPCR. It was shown that the standard deviation of $\mathrm{Ct}$ values for these genes in cDNA of the five single hyphae was larger than those found in biological replicates of cDNA from pools of 100 tips or from
cDNA from the whole periphery. The different yields of RNA obtained from the three sample types (that is, single hyphae, pools of 100 hyphae, and mycelium of the periphery) may have contributed to the variation in standard deviation of the $\mathrm{Ct}$ values. However, the conclusion that the RNA composition between individual hyphae is heterogeneous still holds and is supported by the microarray data of this study as well as our previous findings with GFP reporter studies and in situ hybridizations $[10,11,17]$.

The apical region from which the transcriptome was analyzed represented half of the first hyphal compartment of the exploring hyphae of the colony. Acridine orange staining [14] and in situ hybridizations using $18 S$ rRNA as a probe $[11,17]$ indicate that the selected apical region represents the part of the colony that is most rich in RNA. A high concentration of RNA has been associated with a high growth rate in S. cerevisiae [25]. Levels of rRNA, ribosomal proteins and ribosomes rapidly change when the growth rate of $S$. cerevisiae and Neurospora crassa changes [25-29]. This study showed that the composition of the RNA pool at the hyphal tip is heterogeneous. This heterogeneity does not seem to affect hyphal extension but does have an effect on protein secretion and possibly other cellular activities as well $[10,11]$.

\section{Conclusions}

We performed the first single cell transcriptome analysis of a microbe. It is shown that hyphae that experience identical environmental conditions are heterogeneous with respect to RNA composition. It is thus demonstrated that there is a disconnection between the average gene expression of the hyphae in a particular zone of a colony and the gene expression of a single hypha within such a zone. Therefore, single cell transcriptome analysis with high spatiotemporal resolution should be performed to give an accurate understanding of the processes within a cell. The RNA extraction and amplification protocol that we have developed can be used to provide such expression profiles of single hyphae (or parts thereof) that grow saprobically or that have established a parasitic or mutually beneficial symbiosis with another organism.

\section{Materials and methods}

\section{Strain and growth conditions}

Strain AR9\#2 of $A$. niger was used in this study. This strain is a derivative of strain AB4.1 (pyrG, cspA1) [30] in which the construct pAN52-10S65TGGPn/s was introduced [31]. This construct contains $s G F P(\mathrm{~S} 65 \mathrm{~T})$ under the regulation of the glaA promoter of $A$. niger. To visualize nuclei, CB-A119.1 was used. This strain is a derivative of $\mathrm{N} 593$ (pyrA, cspA) [32] in which construct 
pCB034 was introduced according to previously described protocols [33]. Construct pCB034 contains $s G F P(\mathrm{~S} 65 \mathrm{~T})$ fused to $H 2 B$ under the regulation of the constitutive gpdA promoter. Strains were cultured as a sandwiched colony at $30^{\circ} \mathrm{C}$ in the light. To this end, the fungus was grown between a perforated polycarbonate (PC) membrane (diameter $76 \mathrm{~mm}$, pore size $0.1 \mu \mathrm{m}$; Osmonics, GE Water Technologies, Trevose, PA, USA) and a Lumox membrane (diameter $76 \mathrm{~mm}$; Greiner BioOne, Frickenhausen, Germany) [11]. The PC membrane was placed on top of solidified ( $1.5 \%$ agar) minimal medium [34] containing $25 \mathrm{mM}$ maltose as a carbon source. Freshly harvested spores $(1.5 \mu \mathrm{l}$ of a solution of $0.8 \% \mathrm{NaCl}$ and $0.005 \%$ Tween- 80 containing $10^{8}$ spores $\mathrm{ml}^{-1}$ ) were placed in the center of the PC membrane. The droplet was allowed to dry, after which the Lumox membrane was placed on top of the PC membrane with its hydrophobic side facing the inoculum.

\section{Calcufluor white staining and GFP fluorescence}

After removing the Lumox membrane from the sandwiched colony, part of the periphery of the mycelium (with its underlying membrane) was cut with a scalpel and transferred to a glass slide. In the case of calcafluor white (CFW) staining, the sample was fixed with $70 \%$ ethanol and dried at room temperature. CFW staining was done using PBS containing 0.01\% Fluorescent Brightener 28 (Sigma F-3543, St Louis, MO, USA). After staining for 1 minute, the sample was washed once with PBS. For monitoring of GFP fluorescence, samples were submerged in 90\% glycerol in PBS. A Zeiss Axioscope 2PLUS (Carl Zeiss, Germany) equipped with a HBO $100 \mathrm{~W}$ mercury lamp, a Leica LFC 420 C camera $(2,592 \times 1,944$ pixels $)$ and standard DAPI and FITC filters was used to monitor fluorescence of CFW and GFP, respectively. Images were handled with Leica Application Suite software (v.2.8.1).

\section{Laser micro-dissection and pressure catapulting}

After removing the Lumox membrane from the sandwiched colony, the mycelium and the underlying PC membrane were cut with a scalpel and part of the periphery of the colony was placed upside down onto a nucleotide and RNAse free glass slide. The PC membrane, now facing the air, was removed and the mycelium was fixed with $70 \%$ ethanol and air dried. The hyphal tips were isolated by laser pressure catapulting (LPC) using the PALM CombiSystem (Carl Zeiss MicroImaging, Germany) (Additional file 1). This system was equipped with an Axiovert $200 \mathrm{M}$ Zeiss inverted microscope (Carl Zeiss, Germany) and a 3CCD color camera (HV-D30, Hitachi Kokusai Electric, Japan). The PALM CombiSystem was operated with PALM RoboSoftware v.4.0 (Carl Zeiss MicroImaging, Germany). The autoLPC option was routinely used in combination with a $40 \times$ objective. Hyphal material was catapulted into lids of 0.5 $\mathrm{ml}$ Eppendorf tubes that contained $50 \mu \mathrm{l}$ RNAlater (Qiagen, Hilden, Germany).

\section{RNA isolation and amplification}

Three types of RNA samples were isolated from 7-dayold sandwiched colonies of $A$. niger. First, five samples were obtained each containing the RNA from a single hyphal tip; to this end, neighboring hyphae were selected. Second, three samples were obtained each containing the RNA of a pool of 100 neighboring hyphal tips; different colonies were used for this biological triplicate. Third, three samples were obtained from the outer 3-mm region of the sandwiched colony. Each sample was obtained from a different colony. RNA was isolated from these 11 samples. To this end, hyphal material that was collected in $50 \mu \mathrm{l}$ RNAlater was transferred to a 2-ml Eppendorf tube by a quick centrifugation step (Additional file 1). After snap-freezing in liquid nitrogen, two pre-cooled metal bullets $(4.76 \mathrm{~mm}$ in diameter) were added and samples were ground in a Micro-Dismembrator U (B Braun Biotech, Melsungen, Germany) in a chilled container at 1,500 rpm for $60 \mathrm{~s}$. The frozen material was taken up in $250 \mu \mathrm{l}$ Trizol Reagent (Invitrogen, Carlsbad, CA, USA) by vortexing. After removing the metal bullets, $200 \mu \mathrm{l}$ chloroform was added. After mixing well, samples were centrifuged at $10,000 \mathrm{~g}$ for 10 minutes. The water phase (approximately $200 \mu \mathrm{l}$ ) was mixed with $700 \mu \mathrm{l}$ RLT from the RNeasy MinElute Cleanup Kit (Qiagen) to which 143 mM $\beta$-mercaptoethanol was added. RNA was purified following the instructions of the manufacturer and eluted with $12 \mu \mathrm{l}$ RNAse free water. RNA samples were amplified using the WT-Ovation One-Direct RNA Amplification System (Nugen). The quality and quantity of the cDNA samples were checked using a Bioanalyzer (Agilent Technologies) and a Nanodrop (Nanodrop Technologies, Wilmington, DE, USA), respectively.

\section{Quantification of RNA in exploring hyphae}

RNA from 1,000 tips of exploring hyphae (3 to $4 \mu \mathrm{m}$ in width and $100 \mu \mathrm{m}$ in length) was spotted onto a RotiNylon plus membrane (Roth, Karlsruhe, Germany) together with a series of RNA with known concentration. After cross-linking with UV light, the RNA was hybridized overnight at $42^{\circ} \mathrm{C}$ [35] with $\alpha-{ }^{32} \mathrm{P}-\mathrm{CTP}$ labeled random primed probe of $18 \mathrm{~S}$ rDNA. The blot was exposed to X-OMAT Blue XB films (Kodak, NY, USA) in a BioMax cassette with a BioMax TranScreen$\mathrm{HE}$ (Kodak) at $-80^{\circ} \mathrm{C}$.

\section{QPCR analysis on amplified samples}

QPCR was performed using the ABI Prism 7900HT SDS and SYBR Green chemistry (Applied Biosystems, 
Carlsbad, CA, USA). Ct levels were measured for $18 \mathrm{~S}$ rRNA and for mRNA of the glaA and actin gene. Primers were designed according to the recommendations of the PCR master-mix manufacturer (Applied Biosystems). Ct levels of actin were determined with the primers QPCRactFW1 (GTTGCTGCTCTCGTCATT) and QPCRactRV1 (AACCGGCCTTGCACATA) and those of $18 S$ rRNA were determined with primers QPCR 18SFW1 (GGCTCCTTGGTGAATCATAAT) and QP CR18SRV1 (CTCCGGAATCGAACCCTAAT). These products had an amplification efficiency of 2. cDNA of glaA was amplified using primers QPCRglaAFW3 (GCACCAGTACGTCATCAA) and QPCRglaARV3 (GTAGCTGTCAGATCGAAAGT) with an amplification efficiency of 1.98. QPCR reactions were performed using $1 \mathrm{ng}$ cDNA of samples amplified from RNA extracted from single hyphae (1 pg RNA), 100 hyphae (100 pg RNA) and peripheral mycelium from which the RNA was diluted towards 5 pg RNA prior to amplification.

\section{Microarray analysis}

Amplified cDNA (5 $\mu$ g; see above) was fragmented via combined chemical and enzymatic fragmentation using the protocol of the Encore Biotin Module (Nugen). The fragments were biotin-labeled to the 3-hydroxyl end using the same module following the instructions of the manufacturer. The labeled cDNA was hybridized to Affymetrix GeneChip A. niger Genome Arrays. The GeneChip Hybridization, Wash and Stain Kit (Affymetrix) was used for the hybridizations according to the protocol of the manufacturer with the modification that the hybridization cocktail was prepared according to the Encore Biotin Module and that the hybridization time was extended to 40 hours as recommended by Nugen. The MAS5.0 algorithm (Affymetrix) was used for quality control of the hybridized arrays. Summarized expression values of the single hypha samples were calculated using log-scale RMA [36]. The array data have been deposited in NCBI's Gene Expression Omnibus [37] and are accessible through series accession number [GEO:GSE25497] [38].

RNA was extracted in two batches (that is, hyphae 1 to 3 and hyphae 4 and 5). The batch effect on the expression values of individual genes was inferred using a standard model II ANOVA [39]. After correcting the P-values for false discoveries [40], it was found that all perfect match probes showed a significant batch effect with q-values $\leq$ 0.05 before normalization. After background subtraction, normalization and summarization, $2 \%$ of the probe sets showed a batch effect with $P$-values $\leq 0.01$. After false discovery rate correction only three probe sets showed a batch effect with q-values $\leq 0.05$. Taken together, it is concluded that the batch effect was negligible in the normalized data.

PCA and hierarchical clustering of probe sets and samples was performed on the Z-scores (equal to
(Value-Average)/Standard deviation) derived from the log2 RMA data. Hierarchical clustering was performed in Spotfire Decision Site 7.3 software [41] using complete linkage as clustering method and 1-Pearson correlation as distance measure (d). Different subsets were tested for overrepresentation of FunCats [42] and nonFunCat categories with all 14,455 probe sets as background using a hyper-geometrical test [43]. The classification of the $A$. niger genes in FunCat categories has been described [44].

\section{Additional material}

Additional file 1: A protocol describing the procedure to extract and amplify RNA of single hyphae (or parts thereof).

Additional file 2: A figure showing the amount of RNA within 1,000 hyphae.

Additional file 3: A table, similar to Table 1listing $\mathrm{Ct}$ values of a QPCR analysis of the two amplification experiments of RNA from single hyphae (hyphae 1 to 3 and 4 and 5)

Additional file 4: A table listing Affymetrix quality control checks after hybridizing amplified cDNA from single hyphal tips.

Additional file 5: Scatter plots that show that genes with an absent call in the single hypha analysis are generally lowly expressed in a transcriptome analysis of a population of hyphae from the same zone of the colony

Additional file 6: A table listing the probe sets that gave a present call in at least one of the five arrays of a single hypha.

Additional file 7: A table listing the overrepresentation of functional gene categories within the probe sets with a present call in each of the individual hyphae.

Additional file 8: A table listing the probe sets with a present call in at least one of the five hyphae and a standard deviation $>0.5$ between the log2 RMA signals of the single hyphae.

Additional file 9: A figure showing $P$-values of the functional gene categories in Table 4after analyzing hyphae 1 to 5 and hyphae 1 to 3 and 4 and 5 separately.

Additional file 10: A table listing the top 100 genes with the highest signal values in each of the five hyphae.

Additional file 11: A table listing the genes with the highest signal values that can be found in the top 100 of $2,3,4$ or 5 out of the 5 single hyphae.

Additional file 12: A table listing the genes with the highest signal values that can be found in the top 100 of only 1 out of the 5 single hyphae.

\section{Abbreviations}

bp: base pair; CFW: calcofluor white; Ct: cycle threshold; GFP: green fluorescent protein; LPC: laser pressure catapulting; PBS: phosphate-buffered saline; PC: polycarbonate; PCA: principal component analysis; QPCR: quantitative polymerase chain reaction; RMA: robust multi-array analysis.

\section{Acknowledgements}

This work was supported by the Dutch Technology Foundation STW, Applied Science division of NWO and the Technology Program of the Ministry of Economic Affairs.

\section{Author details}

${ }^{1}$ Microbiology and Kluyver Centre for Genomics of Industrial Fermentations, Institute of Biomembranes, Utrecht University, Padualaan 8, $3584 \mathrm{CH}$ Utrecht, The Netherlands. ${ }^{2}$ Microarray Department and Integrative Bioinformatics 
Unit, Swammerdam Institute for Life Sciences, University of Amsterdam, Science Park 904, 1098 XH Amsterdam, The Netherlands. ${ }^{3}$ Netherlands Bioinformatics Centre (NBIC), Geert Grooteplein 28, 6525 GA Nijmegen Nijmegen, the Netherlands.

\section{Authors' contributions}

$\mathrm{CdB}$ designed and performed the experiments. $\mathrm{CdB}, \mathrm{OB}, \mathrm{MJJ}, \mathrm{TMB}$, and HABW analyzed the results. All authors were involved in drafting the manuscript and read and approved its final version.

\section{Competing interests}

The authors declare that they have no competing interests.

Received: 30 November 2010 Accepted: 4 August 2011

Published: 4 August 2011

\section{References}

1. Nobile CJ, Mitchell AP: Microbial biofilms: e pluribus unum. Curr Biol 2007, 17:349-353.

2. Veening J, Smits WK, Kuipers OP: Bistability, epigenetics, and bet-hedging in bacteria. Annu Rev Microbiol 2008, 62:193-210.

3. Wösten HAB, Moukha SM, Sietsma JH, Wessels JGH: Localization of growth and secretion of proteins in Aspergillus niger. J Gen Microbiol 1991, 137:2017-2023.

4. Moukha SM, Wösten HAB, Asther M, Wessels JGH: In situ localization of the secretion of lignin peroxidases in colonies of Phanerochaete chrysosporium using a sandwiched mode of culture. J Gen Microbiol 1993, 139:969-978.

5. Levin AM, de Vries RP, Wösten HAB: Localization of protein secretion in fungal colonies using a novel culturing technique; the ring-plate system. J Microbiol Methods 2007, 69:399-401.

6. Masai K, Maruyama J, Sakamoto K, Nakajima H, Akita O, Kitamoto K: Squareplate culture method allows detection of differential gene expression and screening of novel, region-specific genes in Aspergillus oryzae. Appl Microbiol Biotechnol 2006, 71:881-891.

7. Levin AM, de Vries RP, Conesa A, de Bekker C, Talon M, Menke HH, van Peij NN, Wösten HAB: Spatial differentiation in the vegetative mycelium of Aspergillus niger. Eukaryot Cell 2007, 6:2311-2322.

8. Kasuga T, Glass NL: Dissecting colony development of Neurospora crassa using mRNA profiling and comparative genomics approach. Eukaryot Cell 2008, 7:1549-1564.

9. de Bekker C, van Veluw GJ, Vinck A, Wiebenga LA, Wösten HAB: Heterogeneity of Aspergillus niger microcolonies in liquid shaken cultures. Appl Environ Microbiol 2011, 77:1263-1267.

10. Vinck A, Terlou M, Pestman WR, Martens EP, Ram AF, van den Hondel CAMJJ, Wösten HAB: Hyphal differentiation in the exploring mycelium of Aspergillus niger. Mol Microbiol 2005, 58:693-699.

11. Vinck A, de Bekker C, Ossin A, Ohm RA, de Vries RP, Wösten HAB: Heterogenic expression of genes encoding secreted proteins at the periphery of Aspergillus niger colonies. Environ Microbiol 2011, 13:216-225.

12. Tietjen I, Rihel JM, Cao Y, Koentges G, Zakhary L, Dulac C: Single-cell transcriptional analysis of neuronal progenitors. Neuron 2003, 38:161-175.

13. Tang F, Barbacioru C, Wang Y, Nordman E, Lee C, Xu N, Wang X, Bodeau J, Tuch BB, Siddiqui A, Lao K, Surani MA: mRNA-Seq whole-transcriptome analysis of a single cell. Nat Methods 2009, 6:377-382.

14. de Bekker C: Hyphal heterogeneity in Aspergillus niger. PhD thesis University of Utrecht, Science Faculty; 2010.

15. Toriello NM, Douglas ES, Thaitrong N, Hsiao SC, Francis MB, Bertozzi CR, Mathies RA: Integrated microfluidic bioprocessor for single-cell gene expression analysis. Proc Natl Acad Sci USA 2008, 105:20173-20178.

16. Wang $D$, Bodovitz $\mathrm{S}$ : Single cell analysis: the new frontier in 'omics'. Trends Biotechnol 2010, 28:281-290.

17. Teertstra WR, Lugones $L G$, Wösten HAB: In situ hybridisation in filamentous fungi using peptide nucleic acid probes. Fungal Genet Biol 2004, 41:1099-1103.

18. De Souza CP, Osmani AH, Hashmi SB, Osmani SA: Partial nuclear pore complex disassembly during closed mitosis in Aspergillus nidulans. Curr Biol 2004, 14:1973-1984.

19. Goldstein L: Stable nuclear RNA returns to post-division nuclei following release to cytoplasm during mitosis. Exp Cell Res 1974, 89:421-425.
20. Alberts B, Bray D, Lewis J, Raff M, Roberts K, Watson JD: Molecular Biology of the Cell. 3 edition. New York: Garland Publishing; 1994.

21. Pang $\mathrm{H}$, Winkler $\mathrm{HH}$ : The concentrations of stable RNA and ribosomes in Rickettsia prowazekii. Mol Microbiol 1994, 12:115-120.

22. Zenklusen D, Larson DR, Singer RH: Single-RNA counting reveals alternative modes of gene expression in yeast. Nat Struct Mol Biol 2008, 15:1263-1271.

23. Neidhardt FC, Umbarger HE: Chemical composition of Escherichia coli. In Escherichia coli and Salmonella. Edited by: Neidhardt FC. Washington, Washington DC: ASM Press; 1996:13-16.

24. Clément-Ziza M, Gentien D, Lyonnet S, Thiery J, Besmond C, Decraene C: Evaluation of methods for amplification of picogram amounts of total RNA for whole genome expression profiling. BMC Genomics 2009, 10:246.

25. Waldron C, Lacroute F: Effect of growth rate on the amounts of ribosomal and transfer ribonucleic acids in yeast. J Bacteriol 1975, 122:855-865.

26. Sturani E, Magnani F, Alberghina FAM: Inhibition of ribosomal RNA synthesis during a shift down transition of growth in Neurospora crassa. Biochim Biophys Acta 1973, 319:153-164.

27. Sturani E, Costantini MG, Zippel R, Alberghina FAM: Regulation of RNA synthesis in Neurospora crassa. An analysis of a shift-up. Exp Cell Res 1976, 99:245-252.

28. Donovan DM, Pearson NJ: Transcriptional regulation of ribosomal proteins during a nutritional upshift in Saccharomyces cerevisiae. Mol Cell Biol 1986, 6:2429-2435.

29. Herruer MH, Mager WH, Woudt LP, Nieuwint RT, Wassenaar GM, Groeneveld P, Planta RJ: Transcriptional control of yeast ribosomal protein synthesis during carbon-source upshift. Nucleic Acids Res 1987, 15:10133-10144.

30. van Hartingsveldt W, Mattern IE, van Zeijl CMJ: Development of a homologous transformation system for Aspergillus niger based on the pyrG gene. Mol Gen Genet 1987, 206:71-75.

31. Siedenberg D, Mestric S, Ganzlin M, Schmidt M, Punt PJ, van den Hondel CAMJJ, Rinas U: GlaA promoter controlled production of a mutant green fluorescent protein (S65T) by recombinant Aspergillus niger during growth on defined medium in batch and fed-batch cultures. Biotechnol Prog 1999, 15:43-50.

32. Goosen T, Bloemenheuvel G, Gysler C, de Bie DA, van den Broek HJW Swart K: Transformation of Aspergillus niger using the homologous orotidine-5'-phosphate-decarboxylase gene. Curr Genet 1987, 11:499-503.

33. de Bekker C, Wiebenga LA, Aguilar G, Wösten HAB: An enzyme cocktail for efficient protoplast formation in Aspergillus niger. J Mircobiol Methods 2009, 76:305-306.

34. de Vries RP, Burgers K, van de Vondervoort PJl, Frisvad JC, Samson RA, Visser J: A new black Aspergillus species, A. vadensis, is a promising host for homologous and heterologous protein production. Appl Environ Microbiol 2004, 70:3954-3959.

35. de Vries RP, van de Vondervoort PJl, Hendriks L, van de Belt M, Visser J: Regulation of the a-glucuronidase-encoding gene (aguA) from Aspergillus niger. Mol Genet Genomics 2002, 268:96-102.

36. Irizarry RA, Bolstad BM, Collin F, Cope LM, Hobbs B, Speed TP: Summaries of Affymetrix GeneChip probe level data. Nucleic Acids Res 2003, 31:e15.

37. Edgar R, Domrachev M, Lash AE: Gene Expression Omnibus: NCBI gene expression and hybridization array data repository. Nucleic Acids Res 2002, 30:207-210.

38. NCBI's Gene Expression Omnibus. [http://www.ncbi.nlm.nih.gov/geo/].

39. Sokal RR, Rohlf FJ: Biometry: The Principles and Practices of Statistics in Biological Research New York: WH Freeman; 1994.

40. Storey JD, Tibshirani R: Statistical significance for genome-wide studies. Proc Natl Acad Sci USA 2003, 100:9440-9445.

41. Spotfire DecisionSite. [http://spotfire.tibco.com].

42. Ruepp A, Zollner A, Maier D, Albermann K, Hani J, Mokrejs M, Tetko I, Güldener U, Mannhaupt G, Münsterkötter M, Mewes HW: The FunCat, a functional annotation scheme for systematic classification of proteins from whole genomes. Nucleic Acids Res 2004, 32:5539-5545.

43. Draghici S, Khatri P, Martins RP, Ostermeier GC, Krawetz SA: Global functional profiling of gene expression. Genomics 2003, 81:98-104.

44. Pel HJ, de Winde JH, Archer DB, Dyer PS, Hofmann G, Schaap PJ, Turner G, de Vries RP, Albang R, Albermann K, Andersen MR, Bendtsen JD, Benen JA, van den Berg M, Breestraat S, Caddick MX, Contreras R, Cornell M, Coutinho PM, Danchin EG, Debets AJ, Dekker P, van Dijck PW, van Dijk A, 
Dijkhuizen L, Driessen AJ, d'Enfert C, Geysens S, Goosen C, Groot GS, et al: Genome sequencing and analysis of the versatile cell factory Aspergillus niger CBS 513.88. Nat Biotechnol 2007, 25:221-231.

doi:10.1186/gb-2011-12-8-r71

Cite this article as: de Bekker et al:: Single cell transcriptomics of neighboring hyphae of Aspergillus niger. Genome Biology 2011 12:R71.

Submit your next manuscript to BioMed Central and take full advantage of:

- Convenient online submission

- Thorough peer review

- No space constraints or color figure charges

- Immediate publication on acceptance

- Inclusion in PubMed, CAS, Scopus and Google Scholar

- Research which is freely available for redistribution

Submit your manuscript at www.biomedcentral.com/submit

C Biomed Central 